\title{
Adaptación al español de la Substance Dependence Severity Scale: resultados preliminares
}

\author{
Spanish Adaptation of the Substance Dependence \\ Severity Scale: preliminar results
}

\author{
Antonio Vélez-Moreno*; Francisco González-Saiz**; Juan Ramírez López***, Esperanza Torrico \\ Linares*; Fermín Fernández-Calderón****; Antonio J. Rojas*****; Óscar M. Lozano* \\ *Departamento de Psicología Clínica, Experimental y Social. Universidad de Huelva; **Unidad de Salud Mental Comunitaria \\ de Villamartín. Hospital de Salud Mental de Cádiz; ***Servicio Provincial de Drogodependencias de Huelva; ****Agencia de \\ Servicios Sociales y Dependencia de Andalucía. Sevilla; *****Departamento de Psicología. Universidad de Almería
}

\section{Resumen}

La Substance Dependence Severity Scale -SDSS- es una de las pocas escalas que evalúa la dependencia y abuso a sustancias según criterios DSM en términos dimensionales. Diferentes estudios psicométricos han aportado evidencias de validez y fiabilidad en su versión inglesa, aunque no se dispone de una versión en español. El objetivo de este trabajo ha sido describir el proceso de adaptación seguido de la versión inglesa de la SDSS al castellano, y aportar resultados preliminares sobre su fiabilidad y evidencias de validez.

Han participado en el estudio 146 pacientes (79.6\% hombres) admitidos a tratamiento en el Centro Provincial de Drogodependencias de Huelva consumidores de alcohol, cocaína, heroína y cannabis. Además de la SDSS, se administró la EUROPASI y el Test para la Evaluación de la Calidad de Vida en Adictos a Sustancias Psicoactivas -TECVASP-.

La escala de gravedad de la dependencia ha mostrado adecuadas propiedades psicométricas en términos de sus índices de discriminación y de consistencia interna $(\alpha=0.881$ para alcohol; $\alpha=0.814$ para cocaína; $\alpha=0.531$ para cannabis; $\alpha=0.785$ para heroína). En cambio la escala que evalúa el abuso ha mostrado resultados más deficientes. Sobre las evidencias de validez, las evidencias basadas en la estructura interna han mostrado una estructura unidimensional. Además, las evidencias obtenidas basadas en la relación con otras variables apoyan empíricamente las relaciones teóricas postuladas. Los resultados preliminares apoyan el uso de la escala de gravedad de la dependencia. La escala de gravedad que evalúa los criterios de abuso necesita más evidencia empírica para conocer la utilidad de la misma. Por lo tanto, no es aconsejable su uso según la versión actual. Palabras claves: Substance Dependence Severity Scale -SDSS-; adaptación, fiabilidad y validez, gravedad de la dependencia.

\section{Abstract}

The Substance Dependence Severity Scale -SDSS- is one of the few scales that assesses substance dependence and abuse according DSM criteria in dimensional terms. Several studies have provided evidence of psychometric validity and reliability in its English version, but there is no Spanish version available. The aim of this work was to describe the adaptation process of the English version of the SDSS into Spanish, and provide preliminary results on its reliability and validity evidence. Participants were 146 patients (79.6\% male), consumers of alcohol, cocaine, heroin and cannabis admitted to treatment in the Drug Abuse Centre Services of Huelva. Besides the SDSS, the EUROPASI and the Health Related Quality of Life for Drug Abusers test -HRQOLDAwere also administered.

The Substance Dependence Severity Scale -SDSS- has shown adequate psychometric properties in terms of the rates of discrimination and internal consistency ( $\alpha=0.881$ for alcohol; $\alpha=0.814$ for cocaine $\alpha=0.531$ for cannabis; $\alpha=0.785$ for heroin). However, the scale assessing abuse showed poorer results. Concerning the validity evidence, the evidence based on internal structure showed a unidimensional structure. Furthermore, the evidence based from the relationships with other variables empirically support the theoretical relationships postulated. Preliminary results support the use of the Substance Dependence Severity Scale. The severity scale, which evaluates abuse criteria, needs further empirical evidence to assess its utility. Therefore, its current version is not recommended for use.

Keywords: Substance Dependence Severity Scale -SDSS-; adaptation, reliability and validity, severity of dependence. 


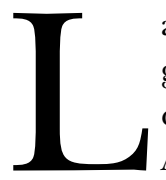
a intervención en los Trastornos por Uso de Drogas (TUS) es compleja pues afecta al ámbito físico, psicológico y social de la vida de los pacientes. Además, frecuentemente cursa con recaídas y de manera crónica (WHO, 2008). Es por ello que su diagnóstico además de determinar la toma de decisiones sobre la elección del tratamiento, debe servir para monitorizar el proceso terapéutico y controlar el impacto del tratamiento y sus resultados en la salud del paciente (Iraurgi-Castillo y González-Saiz, 2002).

Los dos sistemas diagnósticos más utilizados en el ámbito de las drogodependencias son el DSM-IV (APA, 1994) y la CIE-10 (WHO, 1992). Estos sistemas tienen su origen en la definición de Edwards y Gross (Edwards, 1986; Edwards y Gross, 1976) del constructo 'Síndrome de Dependencia Alcohólica' y los elementos que lo comprenden. Estos autores propusieron una descripción empírica de un conjunto de síntomas observables de forma consistente que conforman una estructura sindrómica integrada por síntomas cognitivos, conductuales y fisiológicos relacionados entre sí; y por problemas médicos, sociales y legales relacionados con el alcohol que son conceptual y estadísticamente independientes de la estructura sindrómica (Edwards y Gross, 1976; Edwards et al., 1977; Edwards, 1986; Babor, 1992). Posteriormente, Rounsaville, Spitzer y Williams (1986) propusieron al concepto sindrómico de dependencia de Ewards y Gross (1976) como referente teórico de la adicción, generalizándolo al resto de sustancias psicoactivas. Como consencuencia de ello, en la actualidad ambas nosologías incorporan un diagnóstico de 'dependencia' como conjunto de manifestaciones fisiológicas, comportamentales y cognoscitivas, mientras que las consecuencias adversas del consumo quedan reflejadas en el diagnóstico de 'abuso' del DSM-IV y de 'consumo perjudicial' en la CIE-10. Este sistema de clasificación se ha denominado como sistema categorial, en tanto que delimita al diagnóstico sobre la base de un clúster de síntomas discreto y diferente del clúster de síntomas que definen el otro diagnóstico.

Diferentes escalas y entrevistas diagnósticas han sido desarrolladas a partir de ambas nosologías. En su mayoría, éstas adoptan el enfoque el enfoque categorial de los sistemas de clasificación. Esta aproximación presenta ventajas, como la facilidad para la toma de decisiones terapéuticas con los pacientes fundamentada en la presencia del trastorno, al tiempo que facilita la comunicación entre investigadores y clínicos (Trull y Durret, 2005). Otras escalas, en cambio, han adoptado un modelo de evaluación dimensional, en el que los pacientes se sitúan en un continuo de gravedad de dependencia. Esta perspectiva ha sido incorporada en distintos instrumentos como el Severity of Dependence Questionnaire (Stockwell et al., 1983), el 39-item Alcohol Dependence Data -ADD- (Raistrick, Dunbar y Davidson, 1983), el Severity of Dependence Scale-SDS- (Gossop et al., 1995) o el Leeds Dependence Questionnaire-LDQ- (Raistrick et al., 1994). No obstante, estas escalas no siguen expresamente los criterios fijados por los sistemas nosológicos CIE-10 y DSM-IV, de ahí que sean vistos fundamentalmente como instrumentos de screening.

En la actualidad, aunque el diagnóstico categorial tiene una buena aceptación entre la comunidad clínica e investigadores, cada vez más autores empiezan a señalar la necesidad de disponer de un sistema complementario de evaluación de los pacientes basado en un proceso dimensional. Algunos de los argumentos que avalan su uso son lo siguientes (Kraemer, Noda y O'Hara, 2004; Trull y Durrett, 2005; Helzer et al., 2006): a) existe una amplia hetegeneidad de pacientes cuyas idiosincrasias no quedan adecuadamente visualizadas en el sistema de clasificación categorial; b) una aproximación dimensional es consistente con las investigaciones neurobiológicas que apoyan un proceso gradual de cambio de la actividad celular de los pacientes TUS; c) proporciona una evaluación más comprensiva del estado del paciente y es compatible con la medición categorial; y, d) proporciona ventajas desde una perspectiva estadística.

De entre las escalas que realizan una evaluación de la dependencia en términos dimensionales, la Substance Dependence Severity Scale-SDSS- (Miele et al., 2000a, 2000b) es una escala cuya definición operativa se sustenta en los criterios diagnóstico del DSM y la CIE, lo que la hace diferenciarse de otros instrumentos de estas características. Concretamente, la SDSS es una entrevista clínica semiestructurada desarrollada para evaluar la gravedad de la dependencia actual (últimos 30 días) de una o más sustancias.

Diferentes estudios psicométricos han aportado resultados que avalan su fiabilidad y han aportado evidencias de validez (Miele et al., 2000a; 2000b; 2001), y está incluido entre los instrumentos de evaluación utilizados por el National Drug Abuse Treatment Clinical Trials Network (Rosa, Ghitza y Tai, 2012). A pesar de ello, este instrumento no dispone de una versión española, por lo que el objetivo de este trabajo es describir el proceso de adaptación seguido de la versión inglesa de la SDSS al castellano, y aportar resultados preliminares sobre su fiabilidad y evidencias de validez.

\section{Método}

\section{Participantes}

El marco muestral del estudio está constituido por pacientes con problemas de abuso o dependencia asistentes al Servicio Provincial de Drogodependencias de Huelva (SPDH).

Originariamente se planificó un muestreo aleatorio sistemático entre los pacientes asistentes al SPDH. Sin embargo, debido al escaso número de pacientes captados en los primeros meses, finalmente se optó por aplicar un muestreo accidental. Con éste, el personal asistencial del SPDH ofrecía la posibilidad de participar en el estudio a los pacientes en tratamiento. 
Entre los meses de abril de 2010 y junio de 2012 fueron entrevistados un total de 174 consumidores de diferentes tipos de drogas.

\section{Instrumentos}

La SDSS es una entrevista clínica semiestructurada y heteroadministrada, que tiene como objetivo evaluar la gravedad de la dependencia en términos dimensionales. Dispone de una versión para los criterios de DSM-IV y otra para la CIE-10. Una de las aportaciones frente a otros instrumentos es que la SDSS evalúa, para cada sustancia, dos dimensiones de cada uno de los síntomas del DSM-IV y CIE-10: la frecuencia y la gravedad de los síntomas. Otra de las particularidades que introduce la SDSS es la concreción del marco temporal de evaluación de la gravedad de la dependencia en los 30 días precedentes a la entrevista.

La gravedad de la dependencia es medida a través de 11 ítems. El formato de respuesta para cuatro de estos ítems presenta tres posibles categorías (ausente, sub-umbral, presente). Los siete ítems restantes presentan seis categorías de respuesta en una escala ordinal que va desde la ausencia del síntoma hasta la percepción de éste como muy grave.

La gravedad del abuso también es medida a través de la SDSS. En este caso incluye cuatro ítems que evalúan los cuatro criterios diagnósticos del abuso de sustancias del DSM. Cada ítem consta de seis categorías de respuesta en una escala ordinal, que oscila desde la ausencia del síntoma hasta su percepción como muy grave.

Además de la SDSS, con el objeto de aportar evidencias de validez en un contexto de relaciones teóricas esperables, en este estudio se ha administrado la EUROPASI (Bobes, González, Sáiz, Bousoño, 1996) y el Test para la Evaluación de la Calidad de Vida en Adictos a Sustancias Psicoactivas -TECVASP- (Lozano et al., 2007; 2008; 2009). Este último instrumento ha mostrado sobre la muestra de este estudio un coeficiente de consistencia interna de 0.87 .

Considerando el intervalo temporal de 30 días que establece la SDSS para los diagnósticos de abuso y dependencia, fueron seleccionados cuatro ítems de la entrevista EUROPASI para analizar las relaciones con las puntuaciones de la SDSS: 1) días de tratamiento en el último mes; 2) cantidad de dinero invertido en la sustancia; 3) problemas relacionados con la sustancia; 4) molestia/preocupación por los problemas relacionados con la sustancia. El entrevistado sólo debía referirse a los últimos 30 días seleccionados.

El TECVASP se ha empleado medir la Calidad de Vida Relacionada con la Salud (CVRS). Dicho test está compuesto por 20 ítems de escala ordinal con cinco categorías de respuesta: nada, poco, a veces, bastante, mucho. Puntuaciones bajas en el test indican mala CVRS y puntuaciones altas son indicativas de buena CVRS.

\section{Proceso de adaptación de la SDSS}

La adaptación de la SDSS se ha realizado siguiendo las directrices específicas por la International Test Comission (ITC, 2010) para la adaptación de instrumentos de unas culturas a otras, que incorpora recomendaciones de la adaptación de tests recogidas por Hambleton (2005).

Inicialmente, se analizó la viabilidad de extrapolar este instrumento desde el ámbito norteamericano al español. Por un lado, se constató que la definición operativa de la SDSS se sustenta en el sistema de clasificación DSM-IV (APA, 1994), utilizado también en nuestro ámbito. Además, el estudio realizado por Room et al. (1996) en nueve países -entre ellos EEU y España- encontró que los trastornos por consumo de sustancias definidos por el sistema DSM eran similares en un nivel conceptual. Atendiendo a este estudio y a la amplia implantación en la cultura española del sistema de clasificación DSM, los autores no encontraron motivos para considerar que el constructo de gravedad de la dependencia subyacente a la SDSS no era aplicable al ámbito español.

Posteriormente, el proceso de construcción y adaptación de la SDSS se inició con la traducción al español de la escala por parte de dos personas de manera independiente: una traductora nativa de EEUU asentada en España, y un miembro del equipo de investigación. Una vez realizadas ambas traducciones, los miembros del equipo de investigación valoraron la equivalencia de contenido de cada una de las traducciones con respecto a los criterios diagnósticos del DSM-IV. A continuación se procedió a analizar cada traducción con respecto a la original en términos conceptuales y lingüísticos, valorando la similitud y equivalencia de los ítems. Como resultado de los análisis anteriores, se introdujeron cambios que fueron recogidos en una primera versión de la SDSS, que fue remitida nuevamente a la traductora nativa. Ésta se encargó también de contrastar que los ítems redactados eran conceptual y lingüísticamente semejantes.

Finalmente, los ítems redactados fueron administrados a una muestra de 15 pacientes del SPDH para su pilotaje. Durante dicho proceso se valoró que los ítems fueran correctamente comprendidos por la población objetivo.

\section{Procedimiento}

La administración de la entrevista la realizó un psicólogo clínico, con experiencia en la administración de instrumentos de evaluación. Éste fue entrenado siguiendo las normas del manual de administración de la escala y, antes de iniciar el trabajo de campo propiamente dicho, realizó varias entrevistas de pilotaje.

La entrevista se desarrollaba en una sala del SPDH, donde el entrevistador les informaba sobre el carácter voluntario, le garantizaba el anonimato y su finalidad totalmente ajena al proceso terapéutico que seguían. Una vez que accedía, el entrevistado debía firmar un consentimiento informado 
autorizando al equipo de investigación a utilizar los datos recopilados para su análisis estadístico.

La duración aproximada de la entrevista era de entre 6090 minutos. Al finalizar, el entrevistador entregaba a cada participante un bono de 10 euros para emplear en un supermercado de la ciudad.

\section{Análisis}

La descripción de la muestra se ha realizado sobre los 146 pacientes que forman parte del estudio, calculando los estadísticos descriptivos que proceden de acuerdo a la escala de medida de cada variable.

Los análisis de ítems, fiabilidad y evidencias de validez se han realizado sobre la base de consumidores en los últimos 30 días para las sustancias analizadas. El análisis de ítems comprende las medias y desviaciones típicas, junto a la correlación ítem-total corregida. El análisis de fiabilidad se ha realizado estimando el coeficiente alfa de consistencia interna. En cuanto a las evidencias de validez, se han aportado evidencias basadas en la relación con otras variables y basadas en la estructura interna. Para estas últimas se ha aplicado un análisis factorial con el método de extracción mínimos cuadrados no ponderados y rotación Promin (Lorenzo-Seva, 1999).

Los software empleados han sido SPSS vers. 17 (SPSS, Chicago, 2008) y FACTOR (Lorenzo-Seva y Ferrando, 2006) para realizar el análisis factorial exploratorio como evidencias de la estructura interna.

\section{Resultados}

\section{Descripción de la muestra}

La muestra está formada en su mayoría por hombres (79.6\%). La edad media es de 38.5 (DT=10.2) años, sin observarse diferencias estadísticamente significativas por género. La mayoría son de nacionalidad española $(98.3 \%)$, la media de años de estudios es de 7 (DT=1.8), y un 19,5\% empezó estudios universitarios, de los que sólo un $1.8 \%$ los ha finalizado.

El patrón de empleo durante los últimos tres años releja que un $47.2 \%$ ha estado desempleado habitualmente, un $25 \%$ ha estado trabajando a tiempo completo y un $11.1 \%$ a tiempo parcial. El resto de participantes son estudiantes (4.6\%), jubilados o pensionistas $(5.6 \%)$ o han estado en centros recluidos $(6.5 \%)$.

El patrón de consumo de los participantes refleja que la mayoría han sido policonsumidores. Los indicadores de consumo 'alguna vez' muestran que las sustancias más consumidas han sido cocaína $(89.9 \%)$, alcohol (en grandes cantidades un $87.4 \%)$, cannabis $(82.9 \%)$, heroína $(66.7 \%)$ y benzodiacepinas (24\%). El resto de sustancia se presenta en menores porcentajes. El análisis del indicador de consumo en el 'último mes' muestra resultados más moderados, detectándose que un $60.5 \%$ manifiesta consumo de cannabis, un $51.2 \%$ ha consumido alcohol, un $37.2 \%$ cocaína y un $12.4 \%$ heroína.

Con respecto a la utilización de la vía inyectada, un $23.8 \%$ señala que la ha utilizado en alguna ocasión y sólo un $0.8 \%$ indica que se ha inyectado en los últimos 30 días.

\section{Análisis de ítems y fiabilidad}

En la tabla 1 se presentan las medias y desviaciones típicas, y los índices de discriminación de los 11 ítems que conforman la evaluación de la gravedad de la dependencia a través de la SDSS. Estos resultados son mostrados para las escalas que evalúan dependencia a alcohol, cocaína, cannabis y heroína.

Tabla 1

Medias, desviaciones típicas y correlación ítem-total corregida para los ítems de la escala de dependencia

\begin{tabular}{|c|c|c|c|c|c|c|c|c|}
\hline & \multicolumn{2}{|c|}{ Alcohol } & \multicolumn{2}{|c|}{ Cocaína } & \multicolumn{2}{|c|}{ Cannabis } & \multicolumn{2}{|c|}{ Heroína } \\
\hline & Media (SD) & $\begin{array}{c}\text { Corr. } \\
\text { ítem-total }\end{array}$ & Media (SD) & $\begin{array}{c}\text { Corr. } \\
\text { ítem-total }\end{array}$ & Media (SD) & $\begin{array}{c}\text { Corr. } \\
\text { ítem-total }\end{array}$ & Media (SD) & $\begin{array}{c}\text { Corr. } \\
\text { ítem-total }\end{array}$ \\
\hline D1. Sustancia cantidades superiores & $2.4(1.9)$ & 0.636 & $3.3(1.8)$ & 0.61 & $1.6(1.7)$ & 0.612 & $3.1(1.7)$ & 0.702 \\
\hline D2.A. Deseo persistente & $2.6(1.8)$ & 0.673 & $3.8(1.3)$ & 0.537 & $1.8(1.9)$ & 0.522 & $4.0(1.0)$ & 0.573 \\
\hline D2.B. Intentos infructuosos reducir o controlar & $1.2(0.9)$ & 0.665 & $1.5(0.8)$ & 0.531 & $0.7(0.9)$ & 0.546 & $1.6(0.8)$ & 0.599 \\
\hline D3. Tiempo para obtener/consumir sustancias & $2.9(1.8)$ & 0.701 & $3.5(0.8)$ & 0.452 & $2.5(1.8)$ & 0.650 & $3.3(1.3)$ & 0.440 \\
\hline D4. Reducción actividades sociales & $1.3(1.9)$ & 0.613 & $2.0(2.2)$ & 0.433 & $0.6(1.4)$ & 0.559 & $1.3(1.9)$ & 0.441 \\
\hline D5. Tolerancia & $0.9(1.0)$ & 0.583 & $0.9(1.0)$ & 0.495 & $0.6(0.9)$ & 0.597 & $0.8(1.0)$ & 0.440 \\
\hline D6.A. Síntomas abstinencia & $0.9(1.0)$ & 0.746 & $1.5(0.9)$ & 0.764 & $0.8(0.9)$ & 0.620 & $1.6(0.8)$ & 0.853 \\
\hline D6.B. Experiencia subjetiva gravedad abstinencia & $1.6(1.9)$ & 0.682 & $2.8(1.9)$ & 0.688 & $1.3(1.7)$ & 0.553 & $3.3(1.9)$ & 0.738 \\
\hline D6.C. Consumo para aliviar síntomas abstinencia & $0.8(1.3)$ & 0.688 & $0.9(1.0)$ & 0.244 & $0.6(0.9)$ & 0.755 & $0.8(1.0)$ & 0.042 \\
\hline D7.A. Consumo continuado problemas psicológicos & $1.3(1.6)$ & 0.621 & $2.1(1.7)$ & 0.615 & $0.9(1.6)$ & 0.681 & $1.8(1.9)$ & 0.388 \\
\hline D7.B. Consumo continuado problemas físicos & $0.7(1.5)$ & 0.241 & $0.3(0.9)$ & 0.100 & $0.3(0.9)$ & 0.071 & $0.6(1.3)$ & 0.044 \\
\hline Fiabilidad Alfa de Cronbach & \multicolumn{2}{|c|}{0.881} & \multicolumn{2}{|c|}{0.814} & \multicolumn{2}{|c|}{0.853} & \multicolumn{2}{|c|}{0.785} \\
\hline
\end{tabular}


Se observa que los índices de discriminación de cada ítem con sus respectivas sustancias son, en términos generales, adecuados. No obstante, se aprecia consistentemente que el ítem D7.B sí presenta valores inadecuados, especialmente en el caso de la evaluación de la dependencia a cocaína, cannabis y heroína. Para esta última sustancia también se aprecia que el ítem D.6C presenta un valor bajo.

Por otro lado, la estimación de la fiabilidad calculada a partir del coeficiente alfa de Cronbach aporta resultados adecuados para cada una de las sustancias evaluadas.

En relación a la evaluación de las escalas de abuso de sustancias, los estadísticos descriptivos y el índice de discriminación se muestran en la tabla 3. Se puede apreciar que en las cuatro subescalas los índices estadísticos ponen de manifiesto un comportamiento inadecuado de los ítems, lo que redunda en la obtención de bajos coeficientes de fiabilidad. Así, mientras en los ítems A1 y A3 los índices de discriminación son aceptables, en los otros dos ítems y, especialmente en el A4 (problemas legales) los valores reflejan claramente la ausencia de discriminación.

\section{Evidencias de validez}

En la tabla 3 se muestran los resultados de la contrastación empírica de las relaciones teóricas establecidas en este trabajo que sirven como evidencias de validez. No se han establecido relaciones con las puntuaciones de heroína debido al bajo tamaño muestral.

Se observa que prácticamente todas las relaciones analizadas entre la gravedad del abuso/dependencia y los indicadores de consumo muestran relaciones estadísticamente significativas, en el sentido de que una mayor puntuación en gravedad del abuso/dependencia se relaciona con un mayor número de días de consumo, haber experimentado más problemas y tener una mayor preocupación. No obstante, esta relación no aparece entre los días de consumo de cocaína y las puntuaciones de la escala de abuso, y entre la escala de abuso a cannabis y su preocupación por los problemas experimentados y su preocupación.

Una segunda hipótesis teórica se fundamenta en la relación entre las puntuaciones de gravedad de la dependencia con el dinero gastado durante el último mes. Dicha relación sólo se observa para las escalas de abuso y dependencia de alcohol.

Por otro lado, las relaciones entre la gravedad del abuso/ dependencia y la realización de tratamiento reflejan relaciones negativas. Esto es, un mayor número de días en tratamiento se relaciona con una menor gravedad de las puntuaciones de abuso/dependencia. No obstante, las relaciones sólo son estadísticamente significativas para las escalas de alcohol y cannabis.

Finalmente, se estableció como hipótesis la existencia de relaciones entre la gravedad del abuso/dependencia con la CVRS. Como se refleja en la tabla 3, se observan relaciones negativas que indican que una mayor gravedad del abuso/ dependencia se relaciona con una menor CVRS. Todas las relaciones observadas son estadísticamente significativas, a excepción de la observada entre la CVRS y las puntuaciones de gravedad del abuso.

En relación a las evidencias de validez basadas en la estructura interna, el análisis factorial de las escalas de dependencia a alcohol, cocaína y cannabis mostraron un primer factor con porcentajes de varianzas explicados del $59.9 \%$, $49.5 \%$ y $45.4 \%$ respectivamente. La distribución de los autovalores mostraron una estructura unidimensional, con unas ratios entre el primer y segundo factor de 4.39 en la escala de dependencia a alcohol, 3.89 en la de cocaína y 3.38 en la de cannabis. En relación a los pesos factoriales, como se muestra en la tabla 4, todos los ítems mostraron valores adecuados con la excepción del ítem D7.B en las escalas de dependencia a cocaína y cannabis.

\section{Discusión}

El objetivo de este trabajo ha sido mostrar los resultados preliminares de la adaptación al español de la SDSS, una escala desarrollada en Estados Unidos y de la que aún no se dispone de versión en español, ni para España ni en Latino América. Las características de esta escala, junto a las adecuadas propiedades psicométricas de la versión inglesa han sido los factores conducentes a la adaptación de la misma.

Tal y como plantean los autores de la SDSS, esta escala ha sido diseñada con una doble finalidad: servir como ins-

Tabla 2

Medias, desviaciones típicas y correlación ítem-total corregida para los ítems de la escala de abuso

\begin{tabular}{lcccccccc}
\hline & \multicolumn{2}{c}{ Alcohol } & \multicolumn{2}{c}{ Cocaína } & \multicolumn{2}{c}{ Cannabis } & \multicolumn{2}{c}{ Heroína } \\
\cline { 2 - 9 } & Media (SD) & $\begin{array}{c}\text { Corr. } \\
\text { item-total. }\end{array}$ & Media (SD) & $\begin{array}{c}\text { Corr. } \\
\text { ítem-total }\end{array}$ & Media (SD) & $\begin{array}{c}\text { Corr. } \\
\text { ítem-total }\end{array}$ & $\begin{array}{c}\text { Media (SD) } \\
\text { Corr. } \\
\text { ítem-total }\end{array}$ \\
\hline A1. Incumplimiento de obligaciones & $1.1(1.6)$ & 0.361 & $1.3(1.7)$ & 0.577 & $0.5(1.3)$ & 0.495 & $1.4(1.9)$ & 0.677 \\
A2. Situaciones que comportan peligro físico & $0.7(1.4)$ & 0.108 & $0.9(1.7)$ & 0.344 & $0.9(1.4)$ & 0.144 & $0.5(1.4)$ & 0.356 \\
A3. Problemas sociales/interpersonales & $1.2(1.7)$ & 0.217 & $1.5(1.8)$ & 0.674 & $0.5(1.2)$ & 0.346 & $1.3(1.9)$ & 0.700 \\
A4. Problemas legales & $0.5(0.4)$ & 0.044 & $0.4(1.0)$ & 0.003 & $0.2(0.6)$ & 0.008 & $0.4(0.8)$ & 0.002 \\
\hline Fiabilidad Alfa de Cronbach & \multicolumn{2}{c}{0.345} & & 0.614 & & 0.432 & & 0.645 \\
\hline
\end{tabular}


Tabla 3

Correlaciones entre las puntuaciones de abuso y dependencia con indicadores del último mes

\begin{tabular}{lcccccc}
\hline & $\begin{array}{c}\text { Días Consumo } \\
\text { último mes }\end{array}$ & $\begin{array}{c}\text { Días experimentado } \\
\text { problemas último mes }\end{array}$ & $\begin{array}{c}\text { Preocupación por } \\
\text { consumo último mes }\end{array}$ & $\begin{array}{c}\text { Dinero Gastado } \\
\text { último mes }\end{array}$ & $\begin{array}{c}\text { Días en tratamiento } \\
\text { último mes }\end{array}$ & CVRS \\
\hline $\begin{array}{l}\text { Alcohol } \\
\text { Dependencia }\end{array}$ & $0,309^{*}$ & $0,587^{* *}$ & $0,727^{* *}$ & $0.401^{* *}$ & $-0,295^{*}$ & $-0,553^{* *}$ \\
$\begin{array}{l}\text { Alcohol } \\
\text { Abuso }\end{array}$ & $0,274^{*}$ & $0,607^{* *}$ & $0,327^{*}$ & $0,375^{* *}$ & $-0,383^{* *}$ & $-0,463^{* *}$ \\
$\begin{array}{l}\text { Cocaína } \\
\text { Dependencia }\end{array}$ & $0,343^{*}$ & $0,415^{* *}$ & $0,393^{* *}$ & 0,113 & $-0,162$ & $-0,315^{*}$ \\
$\begin{array}{l}\text { Cocaína } \\
\text { Abuso }\end{array}$ & 0,19 & $0,480^{* *}$ & & & $-0,154$ & $-0,189$ \\
$\begin{array}{l}\text { Cannabis } \\
\text { Dependencia }\end{array}$ & $0,320^{* *}$ & $0,266^{*}$ & $0,334^{*}$ & 0,173 & $-0,327^{* *}$ & $-0,389^{* *}$ \\
$\begin{array}{l}\text { Cannabis } \\
\text { Abuso }\end{array}$ & $0,259^{*}$ & 0,145 & $0,270^{*}$ & $-0,079$ & $-0,375^{* *}$ & $-0,269 *$ \\
\hline
\end{tabular}

*Relación estadísticamente significativa $\mathrm{p}<0.05$

**Relación estadísticamente significativa $\mathrm{p}<0.01$

Tabla 4

Estructura factorial de las escalas de dependencia. Pesos factoriales en primer factor

\begin{tabular}{lccc}
\hline & Alcohol & Cocaína & Cannabis \\
\hline D1. Sustancia cantidades superiores & 0.785 & 0.861 & 0.680 \\
D2.A. Deseo persistente & 0.646 & 0.565 & 0.572 \\
D2.B. Intentos infructuosos reducir o controlar & 0.803 & 0.842 & 0.587 \\
D3. Tiempo para obtener/consumir sustancias & 0.902 & 0.817 & 0.705 \\
D4. Reducción actividades sociales & 0.731 & 0.646 & 0.592 \\
D5. Tolerancia & 0.848 & 0.690 & 0.647 \\
D6.A. Síntomas abstinencia & 0.707 & 0.592 & 0.669 \\
D6.B. Experiencia subjetiva gravedad abstinencia & 0.663 & 0.568 & 0.618 \\
D6.C. Consumo para aliviar síntomas abstinencia & 0.778 & 0.547 & 0.819 \\
D7.A. Consumo continuado problemas psicológicos & 0.861 & 0.791 & 0.739 \\
D7.B. Consumo continuado problemas físicos & 0.421 & 0.236 & 0.095 \\
Prueba de esferecidad de Barlett & $784.3^{* *}$ & $0.624^{* *}$ & $0.412^{* *}$ \\
Prueba KMO & 0.847 & 0.849 & 0.837 \\
\hline
\end{tabular}

**Relación estadísticamente significativa $\mathrm{p}<0.01$

trumento de evaluación inicial de la gravedad de la dependencia, y al mismo tiempo como un indicador de resultado terapéutico sobre este trastorno. Para esta doble finalidad, los autores introdujeron dos cambios con respecto a otras escalas que evalúan el diagnóstico de abuso y dependencia: i) establecieron un sistema de evaluación dimensional, en el que la gravedad de cada ítem es puntuado mediante diferentes alternativas de respuestas; y, ii) establecieron como referencia temporal los últimos 30 días, con el objetivo de poder detectar cambios en la gravedad de la dependencia debido a las intervenciones terapéuticas. Así, los autores consideraron que un marco de 12 meses previos era excesivo para evaluar el cambio en los pacientes y, siguiendo los estudios de McLellan (1992), optaron por fijarlo en los 30 días previos.

Concretando en los resultados obtenidos, y teniendo presente que se trata de resultados preliminares, hasta ahora las conclusiones que se pueden obtener son dispares según se analice la escala de abuso o la de dependencia. Por un lado, la escala formada por los 11 ítems que evalúan la gravedad de la dependencia refleja un comportamiento general adecuado. Diez de los 11 ítems presentan adecuados índices de discriminación, y sólo el ítem relacionado con la aparición de 'problemas físicos a pesar del consumo continuado de la sustancia' refleja valores inapropiados en todas las sustancias. También el ítem relacionado con el 'consumo de sus- 
tancias para evitar los síntomas de abstinencia' muestra un comportamiento inadecuado en la escala de heroína, aunque no en las de alcohol, cocaína y cannabis. Por otro lado, el análisis de la consistencia interna muestra estimaciones del coeficiente alfa apropiados para todas las escalas, al igual que en la versión original (Miele et al., 2000a).

Con respecto a la discriminación de los ítems y la fiabilidad de las escalas de abuso, los resultados encontrados reflejan un comportamiento deficiente para la medición de su gravedad. Como se ha visto, la fiabilidad de la escala aporta valores bajos en términos de consistencia interna. Esto puede venir parcialmente explicado por el reducido número de ítems (Hambleton y Cook, 1983), aunque también puede deberse a factores relacionados con la métrica de los ítems (Elosua y Zumbo, 2008). No obstante, un análisis de la discriminación de cada ítem refleja claramente un comportamiento incorrecto en dos de ellos: 'consumo recurrente en situaciones en las que es físicamente peligroso hacerlo' y 'problemas legales repetidos relacionados con la sustancia'. El primer ítem refleja que no discrimina cuando se evalúa el alcohol y el cannabis, mostrando valores moderados en las escalas de cocaína y de heroína. Este resultado puede estar relacionado con la baja percepción de riesgo asociado al consumo de alcohol y cannabis de la sociedad española (OED, 2010), lo que llevaría a no percibir que bajo el efecto de estas sustancias determinados contextos son peligrosos. El segundo ítem, en cambio, sí puede estar poniendo de manifiesto que existe baja relación entre el constructo que se desea medir y el contenido del ítem. En este sentido, los resultados encontrados apoyan a diversas investigaciones en las que se indica que el criterio diagnóstico de problemas legales no debería formar parte de la evaluación del abuso de sustancias (p.e Langenbucher, Martin, Sanjuán, Bavle y Kirisci, 2004). De hecho, en la planificación del DSM-V se ha excluido el criterio de problemas legales como criterio diagnóstico.

Respecto a las evidencias de validez, siguiendo los Standards de la AERA, APA y NCME (2002), en este estudio se aportan evidencias basadas en la estructura interna de las escalas, y se han establecido un conjunto de hipótesis relacionadas con el constructo de gravedad de la dependencia. En cuanto a las evidencias basadas en la estructura interna, los resultados indican que las tres escalas estudiadas (escalas de dependencia a alcohol, cocaína y cannabis) muestran una estructura esencialmente unidimensional. Y, de manera análoga a lo que ocurre al estudiar el índice de discriminación, el ítem D7.B muestra un comportamiento deficiente dentro del factor de gravedad de la dependencia.

Con respecto a las evidencias basadas en la relación con otras variables, las hipótesis han sido enmarcadas dentro de los últimos 30 días, marco de referencia de la evaluación realizada con la SDSS. Como se ha observado, de manera general, los resultados encontrados apoyan que las puntuaciones derivadas de las diferentes escalas de la SDSS se re- lacionan en los términos teóricos esperados con diferentes variables que forman parte de la red nomológica del constructo de gravedad. Especialmente relevante se consideran las relaciones entre las puntuaciones de la SDSS y el TECVASP, que vienen nuevamente a apoyar empíricamente que una mayor gravedad de la dependencia conlleva una peor calidad de vida relacionada con la salud de los pacientes. Así pues, de manera conjunta, los resultados preliminares de este estudio contribuyen a aportar evidencias basadas en la relación con otras variables, apoyando las puntuaciones que se obtienen de la SDSS.

En cuanto a las limitaciones de los resultados, es necesario tener presente que los resultados que se presentan son preliminares. Considerando los tamaños muestrales, es probable que las escalas de dependencia de cocaína, alcohol y cannabis no sufran demasiadas modificaciones en términos de su fiabilidad y correlación ítem-total corregida, pues como se ha visto, casi todas las correlaciones ítem-total corregida son elevadas. Mayor incertidumbre existe sobre las escalas de abuso, pues al tratarse de muestras aún pequeñas y contener sólo cuatro ítems, es posible que se dé una mayor variabilidad. Con respecto a las relaciones que sirven de apoyo para aportar evidencias de validez, el análisis post-hoc de la potencia del contraste para las correlaciones significativas más débiles se encuentran nuevamente en las escalas de gravedad del abuso de cannabis $(1-\beta)=63.7 \%$, para la de cocaína $(1-\beta)=65.3 \%$ y para la de alcohol $(1-\beta)=61.3 \%$. Para las escalas de dependencia son algo superiores en cada una de las sustancias.

Así pues, a pesar de las limitaciones, este trabajo pone de manifiesto que la adaptación de la SDSS al español presenta inicialmente propiedades psicométricas que pueden avalar su uso. Este hecho, junto a las ventajas a nivel clínico y de investigación de establecer una medida dimensional para el último mes, hace recomendable su utilización por parte de clínicos e investigadores.

\section{Agradecimientos}

El presente trabajo forma parte del proyecto de investigación 'Diagnóstico de gravedad de Dependencia a Sustancias Psicoactivas según criterios DSM-IV. Adaptación al español de la entrevista clínica Substance Dependence Severity Scale (PI10/01155)' financiado por el Instituto de Salud Carlos III y fondos FEDER.

\section{Conflicto de intereses}

No existe conflicto de intereses.

\section{Referencias}

American Educational Research Association, American Psychological Association y National Council on Mea- 
surement in Education. (2002). Standards for educational and psychological testing. Washington D. C: American Educational Research Association.

American Psychiatric Association. (1994). Diagnostic and Statistical Manual of Mental Disorders: DSM-IV. Washington D. C.: American Psychiatric Association.

Babor, T. F. (1992). Substance-related problems in the context of international classificatory systems. En M. Lader, G. Edwards y D.C. Drummond (Eds.). The nature of alcohol and drug related problems. Oxford: Oxford Medical Publications.

Bobes, J., González, M. P., Sáiz, P. A. y Bousoño M. (1996). Índice europeo de severidad de la adicción: EuropASI. Versión española. En Actas de la IV Reunión Interregional de Psiquiatría, 201-218.

Trull, T. J. y Durrett, C. A. (2005). Categorical and dimensional models of personality disorder. Annual Review of Clinical Psychology, 1, 355-380.

Edwards, G. (1986). The alcohol dependence syndrome: a concept as stimulus to enquiry. British Journal of Addiction, 81, 171-183.

Edwards, G. y Gross, M. (1976). Alcohol dependence: provision description of a clinical syndrome. British Medical Journal, 1, 1058-1061.

Edwards, G., Gross, M. M., Keller, M., Moser, J. y Room, R. (1977). Alcohol-related disabilities. WHO Offset Publ. $\mathrm{N}^{\circ}$ 32.Geneve: WHO.

Elosua, P. y Zumbo, B. (2008). Coeficientes de fiabilidad para escalas de respuesta categórica ordenada. Psicothe$m a, 20,896-901$.

Gossop, M., Darke, S., Griffiths, P., Hando, J., Powis, B., Hall, W. y Strang, J. (1995). The Severity of Dependence Scale (SDS): psychometric properties of the SDS in English and Australian samples of heroin, cocaine and amphetamines users, Addiction, 90, 607-614.

Hambleton, R. K. (2005). Issues, designs and technical guidelines for adapting tests into multiple languages and cultures. En R. K. Hambleton, P. F. Merenda y C. D. Spielberger (Eds.). Adapting psychological and educational tests for cross-cultural assessment. Hillsdale, NJ: Erlbaum.

Hambleton, R. K. y Cook, L. L. (1983). Robustness of item response models and effects of test length and sample size on the precision of ability estimates. En D. J. Weiss (Ed.). New horizons in testing (pp. 31-49). New York: Academic.

Helzer, J. E., Van den Brink, W. y Guth, S. E. (2006). Should be both categorical and dimensional criteria for the substance use disorders in DSM-V? Addiction, 101 (suppl. 1), 17-22.

International Test Commission. (2010). International Test Commission Guidelines for Translating and Adapting Tests. [http://www.intestcom.org].

Iraurgi Castillo, I. y González Saiz, F. (2002). Instrumentos de evaluación en drogodependencias. Madrid: Aula Médica.
Kraemer, H. C., Noda, A. y O’Hara, R. (2004). Categorical versus dimensional approaches to diagnosis: methodological challenges. Journal of Psychiatric Research, 38, $17-25$.

Langenbucher, J. W., Labouvie, E., Martin, C. S., sanjuan P. M., Bavly, L. y Kirisci, L. (2004). An application of Item Response Theory Analysis to alcohol, cannabis and cocaine criteria in DSM-IV. Journal of Abnormal Psychology, 113, 72-80.

Lorenzo-Seva, U. y Ferrando, P. J. (2006). FACTOR: A computer program to fit the exploratory factor analysis model. Behavioral Research Methods, Instruments and Computers, 38, 88-91.

Lozano, O. M., Rojas, A. J., Pérez, C., Apraiz, B., Sánchez, F. y Marín A. (2007). Test para la Evaluación de la Calidad de Vida en Adictos a Sustancias Psicoactivas (TECVASP): estudios de fiabilidad y validez. Trastornos Adictivos, 9, 97-107.

Lozano, O. M., Rojas, A. J., Pérez, C., González-Saiz, F., Ballesta, R. y Bilbao I. (2008). Validity evidences of the Health Related Quality of Life for Drug Abuser Test (HRQOLDA Test) based on Biaxial Model of Addiction. Psicothema, 20, 311-317.

Lozano, O. M., Rojas, A. J. y Pérez, C. (2009). Development of a specific Health-Related Quality of Life Test in Drug Abusers using the Rasch Rating Scale Model. European Addiction Research, 15, 63-70.

McLellan, A. T., Kushner, H., Metzger, D., Peters, R., Smith, I., Grissom, G. Pettinati, H. y Argeriou, M. (1992). The Fifth Edition of the Addiction Severity Index. Journal of Substance Abuse Treatment, 9, 199-213.

Miele, G. M., Carpenter, K. M., Cockerman, M. S., Trautman, K. D., Baline, J. y Hasin, D. S. (2000a). Substance Dependence Severity Scale (SDSS): reliability and validity of a clinician-administered interview for DSM-IV substance use disorders. Drug and Alcohol Dependence, 59, 63-75.

Miele, G. M., Carpenter, K. M., Cockerman, M. S., Trautman, K. D., Baline, J. y Hasin, D.S. (2000b). Concurrent and predictive validity of the Substance Dependence Severity Scale (SDSS). Drug and Alcohol Dependence, 59, 77-88.

Miele, G. M., Carpenter, K. M., Cockerman, M. S., Trautman, K. D., Baline, J. y Hasin, D.S. (2001). Substance Dependence Severity Scale reliability and validity for ICD-10 substance use disorders. Addictive Behaviors, 26, 603-612.

Observatorio Español de la Droga y las Toxicomanías. (2011). Situación y tendencia de los problemas de drogas en España. Madrid: Ministerio de Sanidad, Política Social e Igualdad.

Raistrick, D., Bradshaw, J., Tober, G., Weiner, J., Allison, J. y Healey. C. (1994). Development of the Leeds Dependence Questionnaire (LDQ): A questionnaire to mea- 
sure alcohol and opiate dependence in the context of a treatment evaluation package, Addiction, 89, 563-572.

Raistrick, D., Dunbar, G. y Davidson, R. (1983). Development of a questionnaire to measure alcohol dependence, British Journal of Addiction, 78, 89-95.

Room, R., Janca, A., Bennett, L. A., Schmidt, L. y Sartorius, N. (1996). WHO cross-cultural applicability research on diagnosis and assessment of substance use disorders: an overview of methods and selected results. Addiction, 91, 199-220.

Rosa, C., Ghitza, U. y Tai, B. (2012). Selection and utilization of assessment instruments in substance abuse treatment trials: The National Drug Abuse Treatment Clincial Network experience. Substance Abuse Rehabilitation, $17,81-89$

Rounsaville, B. J., Spitzer, R. L. y Williams, J.B. (1986). Proposed changes in DSM-III Substance Use Dirsorders: Description and rationale. American Journal of Psychiatry, 143, 463-468.
Stockwell, T., Murphy, D., Hodgson, R. (1983) The Severity of Alcohol Dependence Questionnaire: Its use, reliability and validity. British Journal of Addiction, 78, 145-155.

Trull, T. y Durret, C. A. (2005). Categorical and dimensional models of personality disorder. Annual Review of Clinical Psychology, 1, 355-380.

World Health Organization. (1992). International Statistical Classification of Diseases and Related Health Problems (ICD10). 1989 Revision. Geneva: World Health Organization.

World Health Organization. (2008). Principles of Drug Dependence Treatment. Discussion Paper. Geneva: World Health Organization. 